\title{
Echocardiographic assessment of rheumatic carditis: a long term follow up study
}

\author{
K S H de Silva ${ }^{1}$, S N Perera $^{2}$, M D Samarasinghe ${ }^{2}$ \\ Journal of the Ceylon College of Physicians, 2012, 43, 20-23
}

\begin{abstract}
Objective: Document the long term cardiac outcome of childhood rheumatic fever (RF).

Method: Children diagnosed with RF were prospectively studied for 5 years from August 1994. Their cardiac status was re-evaluated in 2006 based on WHO criteria.

Results: Initial episode of RF was seen in 91 children during the 5 years from 1994. Fifty (55\%) presented for re-assessment. Six (2 with RC) had discontinued prophylaxis. None had relapses. In the 9 with carditis during the initial presentation, no residual changes were seen in 2, but cardiac damage had progressed in 3 . Three patients initially diagnosed as having mitral valve prolapse (MVP) had evidence of RC. Therefore of the 50 patients re-assessed, 12 (24\%) had evidence of carditis with resolution of changes seen in 2 (16.7\%).
\end{abstract}

Conclusions: Regular assessments with echocardiography are mandatory to confirm and monitor cardiac involvement.

Key words: rhuematic heart disease, echocardiography, Sri Lankan children, long term follow up.

\section{Introduction}

Rheumatic Carditis (RC) is an important cause of acquired heart disease in Sri Lanka. Prophylaxis with benzathine penicillin prevents pharyngitis / tonsillitis due to group A beta-haemolytic streptococci, thereby preventing recurrences of rheumatic fever (RF) and development of chronic rheumatic heart disease ${ }^{1,2,3,4}$.

\footnotetext{
${ }^{1}$ Department of Paediatrics, Lady Ridgeway Childrens Hospital and Faculty of Medicine, University of Colombo.

${ }^{2}$ Department of Cardiology, Lady Ridgeway Childrens Hospital, Colombo.
}

\section{Objectives}

The objective of this study was to document the long term outcome of RC.

\section{Method}

Children with RF, diagnosed according to the revised Jones criteria ${ }^{1}$ admitted to the University Paediatric Unit at Lady Ridgeway Hospital (LRH) from August 1994 to August 1999 were studied. All patients were examined by the chief investigator on admission and thereafter, for appearance of murmurs as carditis evolves over the initial 2 weeks of illness ${ }^{3}$. The condition was discussed in detail with the mother including the importance of adhering to secondary prophylaxis. During hospital stay they were referred for echocardiography to the Cardiology Unit at the National Hospital of Sri Lanka (NHSL). These were performed at NHSL by different cardiologists over the years. The children were regularly seen at LRH till 12 years of age and then referred to the local hospital or NHSL for follow up.

Letters were sent periodically to all our patients starting from July 2006, requesting them to attend the Cardiology Unit at LRH for re-evaluation. Echocardiograms were done over a period of 1 year by 2 Senior Registrars in Paediatric Cardiology. These findings were compared with the findings at initial presentation.

The two paediatric cardiologists involved in the current assessment diagnosed rheumatic carditis based on the presence of the following echocardiography findings as stipulated by the WHO in $2001^{5}$ : dilatation of atria and ventricles, ventricular dysfunction, valvular thickening, failure of coaptation of valve leaflets, restriction of mobility of valve leaflets and valve stenosis or regurgitation. In the mitral valve, thickening of chordae, chordal rupture and thickened subvalvar apparatus were indicative of rheumatic mitral valve involvement. 
Mitral valve prolapse (MVP) had been found in several children at initial presentation. During reassessment MVP was diagnosed in the presence of prolapse of mitral leaflets into the left atrium during systole with or without associated myxomatous degeneration of the mitral valves ${ }^{6}$.

Permission to do the repeat echocardiograms was obtained from the Director, LRH and the Ethical Review Committee of the Faculty of Medicine, Colombo, approved the study.

\section{Results}

There were 91 children with the initial episode of RF during the study period of 5 years. Of these, 50 patients (55\%), 30 of whom were males, responded to our request for re-evaluation. At initial presentation 44 $(88 \%)$ were $>5$ years of age and at re-evaluation 27 (54\%) of them were $>18$ years old - Table 1 . None of them gave a history suggestive of relapses needing hospital admission. Six patients, 2 of them with carditis, had discontinued prophylaxis without medical advice. All others were compliant with secondary prophylaxis.

Table 1. Age distribution of the study population $(n=50)$ at initial presentation and at subsequent evaluation

\begin{tabular}{ccc}
\hline Age (years) & $1994-1999$ & $2006 / 2007$ \\
\hline$<5$ & 6 & - \\
$5-12$ & 44 & 1 \\
$12-18$ & - & 22 \\
$>18$ & - & 27 \\
\hline
\end{tabular}

Tables $2 \mathrm{a}, 2 \mathrm{~b}$ and $2 \mathrm{c}$ are a comparison of the cardiac status of the current study population at initial presentation and subsequent assessment. Of the 18 children with a normal echocardiogram at presentation, 16 continued to have a normal study - Table $2 \mathrm{a}$. Of the 21 initially diagnosed as having MVP, 11 had normal hearts and 3 had evidence of rheumatic carditis at reevaluation - Table 2b. Two patients had isolated trivial mitral regurgitation (without rheumatic heart disease or MVP) which remained same on follow up.
Table 2a. Echocardiographic findings of patients with a normal heart at initial presentation and at subsequent evaluation (Current study population: $n=50$ )

\begin{tabular}{|c|c|c|c|}
\hline \multicolumn{2}{|c|}{$1994-1999$} & \multicolumn{2}{|l|}{$2006 / 2007$} \\
\hline \multirow[t]{2}{*}{ Normal } & $n=18$ & Normal & $n=16$ \\
\hline & & MVP with trivial MR & $\mathrm{n}=2$ \\
\hline
\end{tabular}

Table 2b. Echocardiographic findings of patients with MVP \pm MR at initial presentation and at subsequent evaluation (Current study population: $n=50$ )

\begin{tabular}{lll}
\hline $1994-1999$ & \multicolumn{1}{c}{$2006 / 2007$} & \\
\hline MVP \pm MR $\quad \mathrm{n}=21$ & Normal & $\mathrm{n}=11$ \\
& MVP \pm MR & $\mathrm{n}=6$ \\
& Isolated trivial MR & $\mathrm{n}=1$ \\
& $\begin{array}{l}\text { Rheumatic heart } \\
\text { disease }\end{array}$ & $\mathrm{n}=3$
\end{tabular}

Nine of these 50 patients had rheumatic carditis at initial presentation. The subsequent echocardiograms showed completely normal hearts with no evidence of rheumatic carditis in 2 patients but the changes had progressed in 3, 2 of whom had stopped prophylaxis - Table 2c. Following the current assessment, a total of 12 patients (24\%) had cardiac involvement and in 2 of them (16.7\%), the changes had resolved.

One child with carditis died 3 years after initial presentation with intractable heart failure associated with infective endocarditis. 40 patients: 21 boys and 19 girls did not come for the re-evaluation. At initial presentation, 13 of these patients had normal echocardiograms, 14 had MVP \pm MR and 13 had rheumatic carditis. 
Table 2c. Comparison of the echocardiography findings of patients with rheumatic heart disease at initial presentation $(n=9)$ and at subsequent evaluation, 7 to 12 years later

$1994-1999$

$2006 / 2007$

\section{Resolved}

Grade 1 MR with thickened AMV and restricted mobility of PMV

Rheumatic valvulitis + MVP. No MS/MR

\section{Improved}

Significant MR + mild MS

Grade 2 MR

Grade 1 MR

\section{No change}

Grade 1 MR

\section{Progressed}

Mild MS + trivial MR*

Grade 1-2 MR + mild AR*

Thickened MV and trivial MR

\section{Diagnosed at subsequent assessment}

MVP with myxomatous MV, grade $2 \mathrm{MR}$ MVP with myxomatous MV, grade $2 \mathrm{MR}$ MVP with trivial MR
Normal

Mild MVP with trivial MR. No rheumatic valvulitis

Grade 1 MR, no MS

Grade 1 MR

Rheumatic mitral valvulitis. No MS/MR

Grade 1 MR with thickened AMV

Moderate $\mathrm{MS}+$ trivial MR

Grade 1-2 MR + grade 11 AR

Trivial MR + trivial AR

Rheumatic valvulitis, grade 2-3 MR, no MS Rheumatic valvulitis, grade $1 M R+$ trivial AR Rheumatic valvulitis. No MS/MR/MVP

Total number with rheumatic heart disease in the 50 patients reassessed - 12 (24\%)

AMV - anterior mitral valve, PMV - posterior mitral valve, MVP - mitral valve prolapse

MR - mitral regurgitation, MS - mitral stenosis, AR - aortic regurgitation

* Stopped prophylaxis

\section{Discussion}

The only clinical manifestation of RF which causes permanent damage is carditis ${ }^{3,5,7}$. When the initial episode causes carditis, any recurrence thereafter will almost invariably result in further damage to the heart ${ }^{2,3,4,5}$. Clinical diagnosis of carditis in an acute attack of RF is based on the presence of a murmur of mitral and/or aortic valve regurgitation ${ }^{3,5,8}$. When the heart is affected, almost $80 \%$ of patients will have features of carditis within the first 2 weeks of onset of illness ${ }^{3}$. But absence of a murmur does not exclude involvement of the heart as sub-clinical carditis without an audible murmur is known to occur which has been demonstrated by echocardiography during the initial episode ${ }^{3,5,8,9}$.
It has been shown that if recurrences are prevented, $70 \%$ of patients with mitral valve involvement during the initial attack will eventually have normal hearts ${ }^{1,2,8,10}$. But if recurrences occur, $>50 \%$ of RC will progress to chronic rheumatic heart disease ${ }^{10}$ with associated long term morbidity and mortality ${ }^{3,4,8}$. Therefore, secondary prophylaxis is recommended to all patients with a previous history of RF to prevent colonization or infection of the upper respiratory tract with group A beta-haemolytic streptococci thereby preventing recurrent attacks of $\mathrm{RF}^{4,5,8}$.

A limitation of this study, as mentioned earlier, was the echocardiograms being done by several cardiologists during the initial presentation. But the follow up studies were done based on the same 
diagnostic criteria ${ }^{5}$ by 2 senior registrars in paediatric cardiology. Of the 21 with MVP in the initial evaluation, $3(14.3 \%)$ had evidence of RHD while the echocardiograms were normal in $11(52.4 \%)$ - Tables 2b and 2c.

The long term outcome of RC in Sri Lankan children and adolescents has not been reported previously. The response rate for re-evaluation being $55 \%$, it is difficult to draw definite conclusions regarding this aspect from the present study. But 12 of the 50 patients (24\%) re-assessed 7-12 years after initial presentation, had evidence of rheumatic heart disease. A possible resolution of the cardiac involvement was seen in 2 of these patients (16.7\%) and an improvement in the valvular damage was perceived in 3 (25\%). The damage had progressed in 3 patients, 2 of whom had stopped prophylaxis (Table 2c). Follow up data from India has reported disappearance of established mitral regurgitation in $15 \%$ of their patients compliant with secondary prophylaxis ${ }^{3}$.

\section{Conclusions}

Secondary prophylaxis is essential to prevent recurrences of RF development of valvular disease. Regular echocardiographic evaluations are recommended to confirm rheumatic aetiology and monitor progression of cardiac involvement.

\section{References}

1. Rheumatic fever and rheumatic heart disease. Report of a WHO Study Group, Geneva 1988. World Health Organization Technical Report Series No: 764.

2. Majeed HA. Acute Rheumatic Fever. Medicine International 1997; 12: 100-6.

3. Juneja R, Tandon R. Rheumatic Carditis: A Reappraisal. Indian Heart Journal 2004; 56: 252-5.

4. Saxena Anita. Treatment of Rheumatic Carditis. Indian Journal of Pediatrics 2002; 69(6): 513-16.

5. Rheumatic fever and rheumatic heart disease. Report of a WHO Expert Consultation, Geneva 2001. World Health Organization Technical Report Series No: 923.

6. Mitral Valve Disease. In: Feigenbaum H, Armstrong WF, Ryan T, eds. Feigenbaum's Echocardiography. 6th ed. Philadelphia: Lippincott, Williams and Wilkins; 2005.

7. Treating acute rheumatic fever. Editorial, BMJ 2003; 327 : 631-2.

8. Stollerman Gene H. Rheumatic fever. Lancet 1997; 349: 935-42.

9. Folger Jr Gordon M, Hajar Rachel, Robida Andrej, et al. Occurrence of valvar heart disease in acute rheumatic fever without evident carditis: colour-flow Doppler identification. British Heart Journal 1992; 67: 434-8.

10. Achutti A, Kaplan EL, Nordet P, et al. Streptococcal Sore Throat, Rheumatic Fever, Rheumatic Heart Disease. A reference for physicians and paramedical personnel 1992; 4-16. 